Nepalese Vet. J. 35:55- 62

\title{
Status of Antibiotic Residues in Poultry Meat of Nepal
}

\author{
M. Prajapati, ${ }^{*}$, E. Ranjit, R. Shrestha, S. P. Shrestha, S. K. Adhikari, D. R. Khanal \\ Animal Health Research Division, Nepal Agricultural Research Council \\ *Corresponding author: prajapatimeera@gmail.com
}

\begin{abstract}
Antibiotic residues in livestock products are the burning issue in animal production as well as public health sector. Imprudent use of antibiotics in poultry production increases the risk of antibiotic resistance thus increasing risk in both animals and humans. To determine the trend of antibiotic residues presence in poultry meat, a study was conducted from June 2016 to May 2017 in Kathmandu, Kaski, and Chitwan districts of Nepal. A total of 92 breast samples of broilers (Kathmandu-30, Kaski-40 and Chitwan-22) were collected randomly from different selling outlets of the study areas and tested for residues of commonly used antibiotic viz. Enrofloxacin (EX), Ciprofloxacin (CIP), Streptomycin (STR) and Chloramphenicol (CHL) using the Enzyme Linked Immunosorbent Assay (ELISA) technique. Out of 92 samples, $57(62 \%)$ samples were found positive for antibiotics residue of which 38\% samples were positive for STR residue, 15.2\% for CIP and 8.7\% for EX. However, none of the samples showed positivity for CHL residue. The level of STR was determined to be higher in all districts in comparison to other antibiotics. The meat samples from Kaski were found to contain higher level of STR (56.1\%) while highest percentage of CIP (16.1\%) was detected in Kathmandu. Also, presence of EX was found highest in Kathmandu compared to other districts. The study concluded that antibiotics residues have been abundantly found in poultry meat which can have serious effect in human health. Hence, to mitigate this, routine antibiotics residues monitoring and surveillance programmes in food animal products should be conducted to ensure the consumer safety.
\end{abstract}

Keywords: Ciprofloxacin, Chloramphenicol, Enrofloxacin, Poultry meat, Streptomycin. 


\section{INTRODUCTION}

Antibiotics are used in animal agriculture especially in poultry industry, not only for therapeutic and prophylactic purposes, but also as a growth promoter to enhance the health and productivity of flocks through feed or drinking water (Donoghue, 2003 and Ahmed, 2016). Currently, approximately 80\% of all food-producing animals receive antimicrobial medication at some point as treatment or prevention of disease or growth promoter (Pavlov et al., 2008). The use of antibiotic drugs in food-producing animals may result in the presence of residues in meat and offal. The consumption of these trace levels of antimicrobial residues in foods from animal origin may have consequences on the indigenous human intestinal microflora which constitutes essential component of human physiology. This flora acts as a barrier against colonization of the gastrointestinal tract by pathogenic bacteria and plays an important role in food digestion (Shankar et al., 2010). The massive use of antibiotics and failure to follow the withdrawal period of drugs leads to the consumption of antibiotics at low levels which may increase the risk of occurrence of microbial drug resistance and causes hypersensitivity reaction and disruption of normal intestinal flora in human (Beyene et al., 2016, Nisha et al., 2008).

Many of the drugs used as growth promoters affects the meat quality resulting in poor eating quality. These substances must be administered only in recommended concentration and their respective withdrawal times must be followed to decrease the potential risk to the consumers' health and to ensure the reduction of their residues in edible tissues to an acceptable level. (Kozarova et al., 2004). Several groups of antibiotics including quinolones and fluoroquinolones are frequently used in veterinary medicine for treatment and prevention of diseases, thereby reducing famers' losses (Omotoso and Omojola, 2015). Specifically, Enrofloxacin (EX), Streptomycin (STR), Ciprofloxacin (CIP) and Chloramphenicol (CHL) have been widely used to treat and cure infections both in human and veterinary medicine. In Nepal, since the poultry sector is commercialized comparatively, huge amount of antibiotics is used in this sector. Due to the short lifespan of broiler, it is likely that the withdrawal period is not followed as required. The antibiotic residues in the bodies of chicken may pose adverse effect to human health and may cause undesirable changes in bowel microflora and induce immunological reaction in susceptible persons (Mahmoudi et al., 2014). There is a perception among consumers that the food supply contains high concentrations of drug or hormone residues causing significant health concerns or problems (Donoghue, 2003). However, very few studies have been conducted in Nepal to estimate the level of antibiotic residues in poultry meat in Nepal. The objective of this was to detect the presence of antibiotic residues in chicken meat samples from three major cities of Nepal. 


\section{MATERIALS AND METHODS}

The meat samples were collected from meat shops of Chitwan, Kaski and Kathmandu from June 2016 to May 2017. A total of ninety-two samples of breast meat from broilers (Kathmandu-30, Kaski-40 and Chitwan-22) were collected randomly from different selling outlets (only one sample from each outlet) in and around selected cities. The outlets were chosen randomly and purposively. About $50 \mathrm{~g}$ of each sample was cut aseptically from the breast meat and transferred in self-sealing colorless polythene bags. The bags were labeled and transported in a cool box containing chiller packs to Animal Health Research Division (AHRD), Nepal Agriculture Research Council, Khumaltar, Kathmandu All the meat samples were stored at $-40{ }^{\circ} \mathrm{C}$ until the time of analysis.

During analysis, the chicken meat samples were thawed overnight in a refrigerator. Each meat sample were cut into small pieces and blended at $2000 \mathrm{rpm}$ in a tissue blender for two minutes. Ten grams of blended meat tissue and $10 \mathrm{ml}$ of deionized water were taken in $50 \mathrm{ml}$ polypropylene centrifuge tube. Extraction method was adopted for sample analysis as per manufacturer's kit (Bioscientific Corporation, USA).

\section{Sample preparation for EX}

The meat sample was homogenized with a mortar and pestle. Then one gram of homogenized sample was kept in four $\mathrm{ml}$ of $35 \%$ Methanol or sample extraction buffer I as provided in the kit. The solution was vortexed at maximum speed and then centrifuged for 10 minutes at $4,000 * \mathrm{~g}$ at room temperature $\left(20-25^{\circ} \mathrm{C}\right)$. Half $\mathrm{ml}$ of the supernatant was transferred to a tube and $25 \mu 1$ of meat extraction buffer II plus $1.5 \mathrm{ml}$ of $35 \%$ Methanol or sample extraction buffer was added and vortexed. After mixing the solution, $50 \mu \mathrm{l}$ of the supernatant was taken for the assay. The sensitivity (detection limit) and specificity (cross reactivity) of EX was one parts per billion (ppb) and 100\% respectively.

\section{Sample preparation for CIP}

Fat from the sample was cleared and homogenized with mortar and pestle. About one $\mathrm{g}$ of homogenized sample was mixed with four $\mathrm{ml}$ of $70 \%$ methanol. The solution was vortexed for 10 minutes at maximum speed. It was then centrifuged for five minutes at $4000^{*} \mathrm{~g}$ at room temperature $\left(20-25^{\circ} \mathrm{C}\right)$. Then $0.5 \mathrm{ml}$ of the supernatant was transferred to a tube and $0.5 \mathrm{ml}$ of $1 \mathrm{X}$ sample extraction buffer was added on it. After mixing the solution, $50 \mu 1$ of the supernatant was taken for the assay. The sensitivity and specificity of CIP was $3.5 \mathrm{ppb}$ and $100 \%$ respectively. 


\section{Sample preparation for STR}

Fat from the sample was cleared and homogenized with mortar and pestle. About 0.5 $\mathrm{g}$ of homogenized sample was mixed with $4.5 \mathrm{ml}$ of $1 \mathrm{X}$ sample extraction buffer and the solution was vortexed for three minutes at maximum speed. Then $0.2 \mathrm{ml}$ of the homogenized sample was taken and centrifuged for five minutes at $4000^{*} \mathrm{~g}$ at room temperature $\left(20-25^{\circ} \mathrm{C}\right)$. Then $0.5 \mathrm{ml}$ of the supernatant was transferred to a new tube and incubated at $75^{\circ} \mathrm{C}$ for five minutes. The solution was vortexed for one minute and then centrifuged for five minutes at $4000^{*} \mathrm{~g}$ at room temperature. The $0.2 \mathrm{ml}$ of the supernatant was transferred to a new tube and mixed with of $5 \mathrm{ul}$ of $1 \mathrm{X}$ sample extraction buffer. The solution was vortexed. $50 \mu 1$ of mixture was used for the assay. The sensitivity and specificity of STR was 5 ppb and 100\% respectively.

\section{Sample preparation for CHL}

Reasonable amount of sample was homogenized with a suitable mixer and about $3 \mathrm{~g}$ of the homogenized sample was mixed with $6 \mathrm{~mL}$ of ethyl acetate. The mixture was then vortexed for $3 \mathrm{~min}$ at maximum speed and centrifuged for $5 \mathrm{mins}$ at $4,000 \mathrm{xg}$ at room temperature $\left(20-25^{\circ} \mathrm{C}\right) .4 \mathrm{~mL}$ of the ethyl acetate supernatant was transferred into a new vial and dried the sample in a $60-70^{\circ} \mathrm{C}$ water bath under reduced pressure. The dried residue was dissolved in $2 \mathrm{~mL}$ of n-hexane in which $1 \mathrm{ml}$ of $1 \mathrm{X}$ sample extraction buffer was added. Then the mixture was vortexed at maximum speed for 2 mins and centrifuged for $10 \mathrm{mins}$ at 4,000 $\mathrm{x} g$ at room temperature. The upper hexane layer was discarded. $100 \mu \mathrm{l}$ of the lower aqueous layer was used for the assay. The sensitivity (Detection Limit) and specificity (Cross Reactivity) of CHL was 0.0075 ppb and $100 \%$ respectively.

\section{Enzyme Linked Immunosorbent Assay (ELISA) test procedure}

The manufacturer's instruction was followed for ELISA (Bioscientific Corporation, USA) testing and the concentration of all the tested antibiotics were calculated. There were separate ELISA test Kits for EX, CIP and STR and CHL antibiotics.

\section{RESULT}

The result of this study indicated that residues of antibiotics EX, STR, CIP and CHL were detected in samples of broiler breast muscles by ELISA. Out of 92 chicken meat samples, 57 (62\%) samples were found positive for antibiotics residue of which 35 samples (38\%) were positive to STR residue, 14 (15.21\%) samples were positive to CIP, $8(8.7 \%)$ samples were found positive for EX while none of the samples were positive for CHL (Figure 1). 


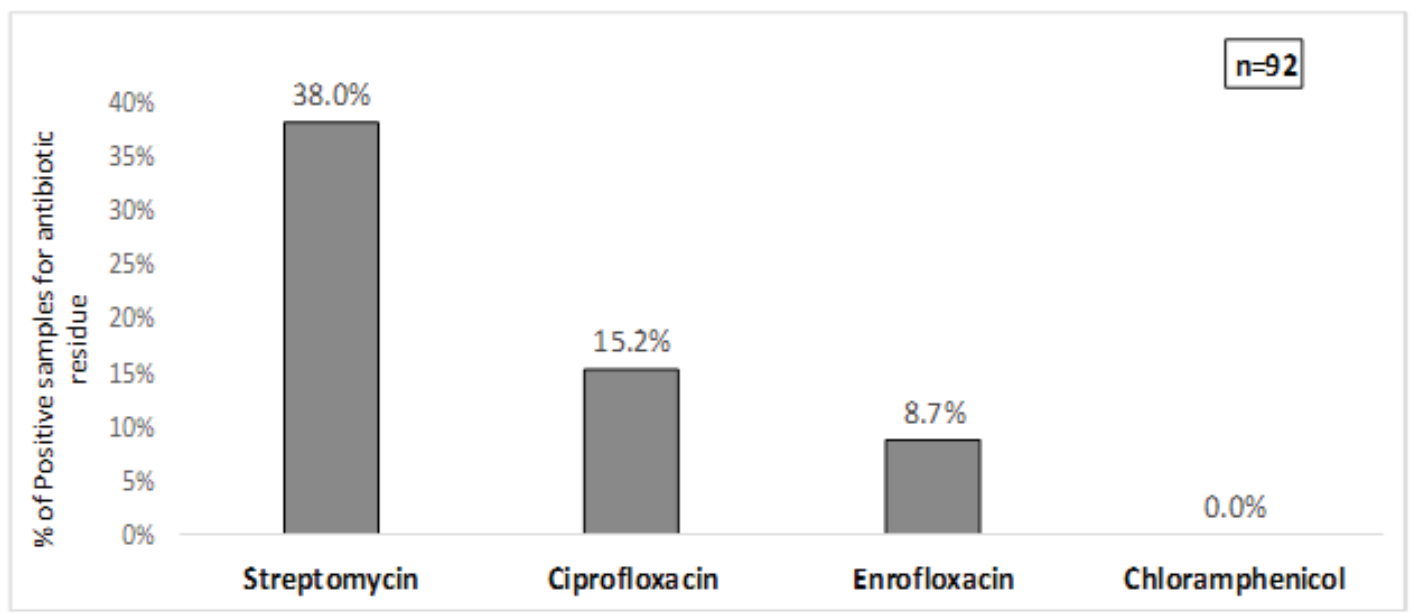

Fig. 1: Positive meat samples for antibiotic residue.

The presence of STR was determined in $57.5 \%$ of the meat samples from Kaski whereas only $30.0 \%$ of samples from Kathmandu and $13.6 \%$ of samples from Chitwan showed the presence of STR residue. In case of CIP, $16.7 \%$ of meat samples collected from Kathmandu was found to be positive whereas only $15.0 \%$ of samples from Kaski and $13.6 \%$ of samples from Chitwan showed the presence of CIP. Likewise, $13.3 \%$ of meat samples from Kathmandu was found with residues of CX.

The CHL was not detected in samples from any districts (Figure 2).

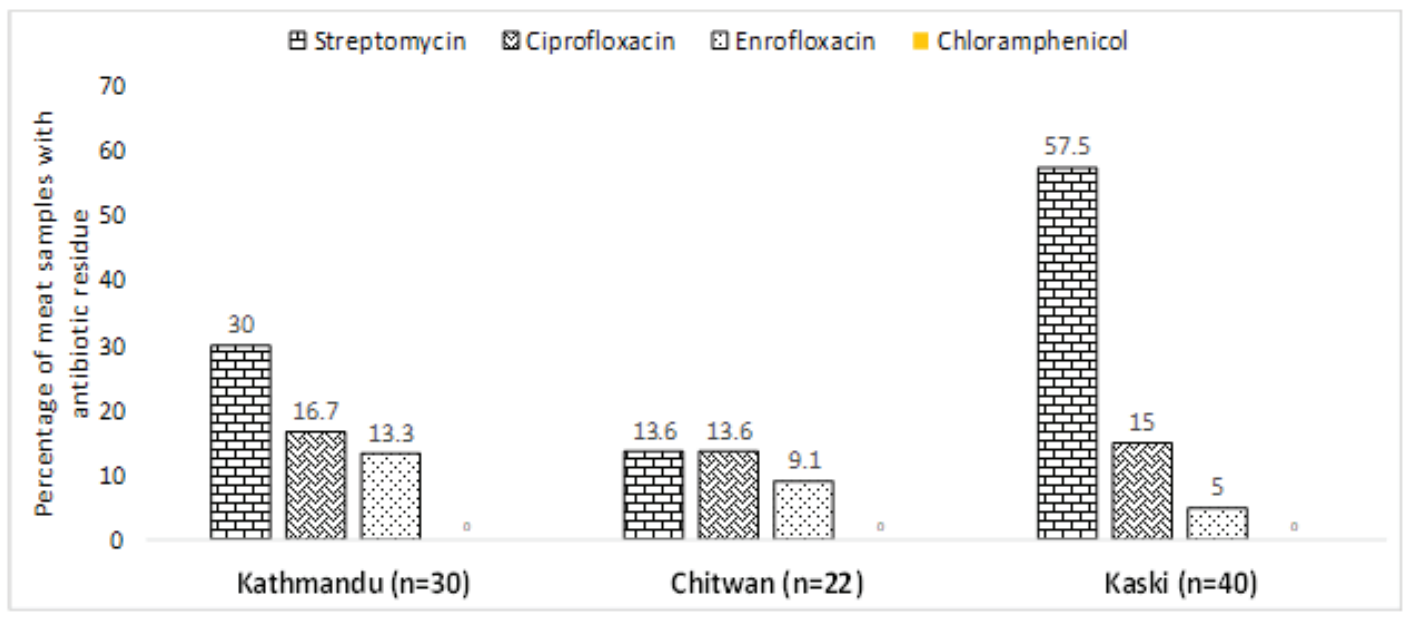

Fig. 2: Positive meat samples for antibiotic residues on the basis of location. 


\section{DISCUSSION}

In the present study, out of 92 samples assayed by ELISA, 8 (8.7\%), 35 (38.0\%), $14(15.2 \%)$ and none had detectable levels for EX, STR, CIP and CHL residues respectively. Similarly, higher level of CIP (50\%) residues were found in a poultry meat in a similar study conducted in Nigeria (Omotoso and Omojola, 2015). The similarity in the result might be due to frequently administered of Fluoroquinolones in poultry rearing in Nepal and Nigeria. The CIP is also a marker residue for EX that is approved for use as a veterinary drug and when it is metabolized, its pharmacologically active metabolite, CIP, is produced (Tyczkowska, et al., 1989) The detection of CIP during market screening often reflects the use of both EX and CIP (Navratilova et al., 2011; Botsoglou and Fletouris, 2001).

The present study revealed that the concentration of STR was found higher than other antibiotics in all districts. Samples (57.5\%) from Kaski were found to contain STR residues followed by Kathmandu with $30.0 \%$ of positive samples. The high occurrence of STR may be due to the use of antibiotic in poultry for the preventive measure.

\section{CONCLUSION}

The results of the present study suggest that fluoroquinolones have been abundantly used in poultry. To mitigate this, routine antibiotics residues monitoring and surveillance programmes in food animal products should be conducted to ensure the consumer safety. Awareness regarding the rational use of antibiotics and its serious effects on the human health should be initiated to educate the farmers. The use of rapid screening test along with quantitative methods to establish minimum residual levels of different types of antibiotics in meat along with respecting withdrawal period for antibiotics in food animals need to be established. The misuse of antibiotics in the poultry farm should be discouraged and be used only with veterinarian's prescription.

\section{ACKNOWLEDGEMENT}

We would like to thank all supporting staffs of Animal Health Research Division (AHRD), District Livestock Service Office (DLSO) Chitwan and Pokhara for their help and cooperation during the study. 


\section{REFERENCES}

Ahmed, A. M., \& Gareib, M. M. (2016). Detection of Some Antibiotics Residues in Chicken Meat and Chicken Luncheon. Egyptian Journal of Chemistry and Environmental Health, 2(2): 315-323.

Beyene, T. (2016). Veterinary Drug Residues in Food-animal Products: Its Risk Factors and Potential Effects on Public Health. Journal of Veterinary Science and Technology, 7(1): 285-291. doi: 10.4172/2157-7579.1000285

Botsoglou, N. A., \& Fletouris, D. J. (2001). Drug residues in foods : pharmacology, food safety, and analysis. New York: Marcel Dekker.

Donoghue, D. J. (2003). Antibiotic residues in poultry tissues and eggs: human health concerns? [Review]. Poultry Science, 82(4): 618-621. doi: 10.1093/ $\mathrm{ps} / 82.4 .618$

Kožárová, I., Máté, D., Hussein, K., Raschmanová, K., Marcincak, S., \& Jevinová, P. (2004). High-performance liquid chromatographic determination of sulfadimidine residues in eggs. Acta Veterinaria, 54(5-6). doi: 10.2298/ avb0406427k

Mahmoudi, R., Gajarbeygi, P., Norian, R., \& Farhoodi, K. (2014). Chloramphenicol, sulfonamide and tetracycline residues in cultured rainbow trout meat (Oncorhynchus mykiss). Bulgarian Journal of Veterinary Medicine, 17(2): 147-152.

Navrátilová, P., Borkovcová, I., Vyhnálková, J., \& Vorlová, L. (2018). Fluoroquinolone residues in raw cow's milk. Czech Journal of Food Sciences, 29(6): 641-646. doi: $10.17221 / 22 / 2011$-cjfs

Nisha, A. R. (2008). Antibiotic residues - A global health hazard. Veterinary World, 1(12): 375-377. doi: doi: 10.5455/vetworld.2008.375-377

Omotoso, A. B., \& Omojola, A. (2015). Fluoroquinolone residues in raw meat from open markets in Ibadan, Southwest, Nigeria. International Journal of Health and Animal Science Food Safety, 2(1): 32-40. doi: 10.13130/2283-3927/4739

Pavlov, A., Lashev, L., Vachin, I., \& Rusev, V. (2018). Residues of antimicrobial drugs in chicken meat and offals. Trakia Journal of Sciences, 6(1): 23-25.

Shankar, Manjunatha, P., Shivamallu, C., Ranjith, \& and Shivakumar, D. (2010). 
Rapid Methods for detection of Veterinary Drug residues in Meat. Veterinary World, 3(5): 241-246.

Tyczkowska, K., Hedeen, K. M., Aucoin, D. P., \& Aronson, A. L. (1989). Highperformance liquid chromatographic method for the simultaneous determination of enrofloxacin and its primary metabolite ciprofloxacin in canine serum and prostatic tissue. Journal of Chromatography B: Biomedical Sciences and Applications, 493, 337-346. doi: https://doi.org/10.1016/S03784347(00)82739-5 